\title{
Dispersionless sTB
}

\author{
Ashok Das \\ Department of Physics and Astronomy, \\ University of Rochester, \\ Rochester, NY 14627-0171 \\ USA \\ and \\ Ziemowit Popowicz \\ Institute of Theoretical Physics, \\ University of Wroc/l aw, \\ 50-205 Wroc/l aw \\ Poland.
}

\begin{abstract}
We analyze the dispersionless limits of the SUSY TB-B (sTB-B) and the SUSY TB (sTB) hierarchies. We present the Lax description for each of these models, as well as the $N=2 \mathrm{sTB}$ hierarchy and bring out various properties associated with them. We also discuss open questions that need to be addressed in connection with these models.
\end{abstract}




\section{Introduction:}

In recent years, dispersionless integrable models have received a lot of attention. They involve equations of hydrodynamic type [1-5] and include such systems as the Riemann equation [5-7], the the polytropic gas dynamics [5,8], the chaplygin gas and the Born-Infeld equation [9-10]. These are models which can be obtained from a "classical" limit [3,11] of integrable models where the dispersive terms are absent. They have many interesting properties including the fact that, unlike their dispersive counterparts, each of them can be described by a Lax equation which involves a Lax function in the classical phase space and a classical Poisson bracket relation. Even more interesting and more difficult are the supersymmetric dispersionless models. In a recent paper [12], we gave, for the first time, the Lax description for the dispersionless supersymmetric KdV equation [13] as well as the dispersionless Kupershmidt equation [14]. Unlike the bosonic models, the Lax functions for the dispersionless supersymmetric models do not follow trivially from the Lax operator of the dispersive counterpart. Furthermore, while a lot of the interesting properties follow from the Lax description of the model, we also pointed out several open questions that arise. In this paper, we follow up on our earlier investigation and describe the Lax formulation for the dispersionless supersymmetric two boson (TB) hierarchy.

The TB hierarchy [15] as well as its supersymmetric counterpart [16] are known to yield various other integrable models upon appropriate reduction. In this sense, the supersymmetric TB hierarchy is a more interesting model to study. In particular, it has a natural $N=2$ supersymmetry [16-17] and its dispersionless limit would lead to the first $N=2$ supersymmetric model of its kind. There are, in fact, two distinct supersymmetric generalizations of the TB hierarchy. The first is known as the sTB-B hierarchy [18], so called because it leads upon reduction to the supersymmetric $\mathrm{KdV}$ equation considered by the Beckers [19]. The second supersymmetric generalization, on the other hand, leads upon reduction to the supersymmetric KdV equation considered by Manin and Radul [13]. We call this the sTB hierarchy [16] (although, a more appropriate name may be sTB-MR along the same lines).

In section 2, we give the Lax description for the dispersionless sTB-B hierarchy, which is fairly straightforward, and bring out its properties as well as the open questions associated with this model. In section 3, we give the Lax description for the dispersionless sTB hierarchy. This is quite nontrivial and naturally reduces to the dispersionless sKdV equation upon appropriate restriction. However, unlike the sTB model, it does not lead to the dispersionless supersymmetric non-linear Schrödinger equation. In fact, even the dispersionless bosonic TB model does not quite give the dispersionless non-linear Schrödinger equation (at least, we have not succeeded in finding a field redefinition which would do this). We bring out various properties of the dispersionless sTB model as well as the open questions associated with this system. In section $\mathbf{4}$, we describe the dispersionless

sTB system in a manifestly $N=2$ supersymmetric formulation. Finally, we make some brief observations in section $\mathbf{5}$. We have used REDUCE [27] and special supersymmetric package in Reduce [28] extensively in some of the algebraic calculations. 


\section{Dispersionless Limit of sTB-B Equation:}

The sTB hierarchy [16], like the TB hierarchy, is an integrable system in $1+1$ dimensions. The basic dynamical variables for this system are the two fermionic superfields

$$
\begin{aligned}
& \Phi_{0}(t, x, \theta)=\psi_{0}+\theta J_{0} \\
& \Phi_{1}(t, x, \theta)=\psi_{1}+\theta J_{1}
\end{aligned}
$$

where $\theta$ represents a Grassmann coordinate and we are suppressing the space-time dependence on the right hand side for simplicity. The sTB-B hierarchy is described by the non-standard Lax equation [18]

$$
\frac{\partial L}{\partial t_{n}}=\left[L,\left(L^{n}\right)_{\geq 1}\right]
$$

where $n=1,2, \ldots$ and the Lax operator has the form

$$
L=D^{2}-\left(D \Phi_{0}\right)+D^{-2}\left(D \Phi_{1}\right)
$$

with the super-covariant derivative defined to be

$$
D=\frac{\partial}{\partial \theta}+\theta \frac{\partial}{\partial x}, \quad D^{2}=\frac{\partial}{\partial x}
$$

Explicitly, the first three flows of the sTB-B hierarchy have the form

$$
\begin{gathered}
\frac{\partial \Phi_{0}}{\partial t}=-\Phi_{0 x}, \quad \frac{\partial \Phi_{1}}{\partial t}=-\Phi_{1 x}, \\
\left.\frac{\partial \Phi_{0}}{\partial t}=-\left(D^{4} \Phi_{0}\right)+D\left(\left(D \Phi_{0}\right)^{2}+2\left(D \Phi_{1}\right)\right)\right), \\
\frac{\partial \Phi_{1}}{\partial t}=\left(D^{4} \Phi_{1}\right)+2 D\left(\left(D \Phi_{0}\right)\left(D \Phi_{1}\right)\right), \\
\frac{\partial \Phi_{0}}{\partial t}=-\Phi_{0 x x x}-D\left(6\left(D \Phi_{0}\right)\left(D \Phi_{1}\right)-3\left(D \Phi_{0}\right)\left(D \Phi_{0 x}\right)+\left(D \Phi_{0}\right)^{3}\right), \\
\frac{\partial \Phi_{1}}{\partial t}=-\Phi_{1 x x x}-3 D\left(\left(D \Phi_{1}\right)^{2}+\left(D \Phi_{1}\right)\left(D \Phi_{0}\right)^{2}+\left(D \Phi_{1 x}\right)\left(D \Phi_{0}\right)-2\left(D \Phi_{1}\right)\left(D \Phi_{0 x}\right)\right) .
\end{gathered}
$$

The last two equations lead, under the reduction $\Phi_{0}=0$, to the supersymmetric $\mathrm{KdV}$ equation considered by the Beckers [19] (the so called sKdV-B equation).

$$
\frac{\partial \Phi_{1}}{\partial t}=-\Phi_{1 x x x}-3 D\left(\left(D \Phi_{1}\right)^{2}\right) .
$$

The Lax description for the dispersionless limit of the sTB-B hierarchy is quite straightforward, much like the dispersionless limit of the sKdV-B hierarchy [12]. Consider the Lax function

$$
L=p-\left(D \Phi_{0}\right)+p^{-1}\left(D \Phi_{1}\right)
$$


where $p$ is the momentum variable of the classical phase space, satisfying the canonical $\mathrm{PB}$ relations

$$
\{x, p\}=1, \quad\{x, x\}=0=\{p, p\} .
$$

Then, it is easily seen, with the standard canonical Poisson bracket relations $\left(\{p, f\}=-\frac{d f}{d x}\right)$, that the Lax equation

$$
\frac{\partial L}{\partial t_{n}}=\left\{\left(L^{n}\right)_{\geq 1}, L\right\}
$$

leads to the dispersionless sTB-B hierarchy. Explicitly, the first three flows of this hierarchy are,

$$
\begin{array}{cc}
\frac{\partial \Phi_{0}}{\partial t}=-\Phi_{0 x}, & \frac{\partial \Phi_{1}}{\partial t}=-\Phi_{1 x} \\
\frac{\partial \Phi_{0}}{\partial t}=2\left(D \Phi_{0}\right) \Phi_{0 x}+2 \Phi_{1 x}, & \frac{\partial \Phi_{1}}{\partial t}=2 D\left(\left(D \Phi_{0}\right)\left(D \Phi_{1}\right)\right) \\
\frac{\partial \Phi_{0}}{\partial t}=D\left(-\left(D \Phi_{0}\right)^{3}-6\left(D \Phi_{1}\right)\left(D \Phi_{0}\right)\right) \\
\frac{\partial \Phi_{1}}{\partial t}=-3 D\left(\left(D \Phi_{1}\right)^{2}+\left(D \Phi_{1}\right)\left(D \Phi_{0}\right)^{2}\right)
\end{array}
$$

Equation (14) allows the reduction $\left(\Phi_{0}=0\right)$ to the dispersionless supersymmetric KdV-B equation

$$
\frac{\partial \Phi_{1}}{\partial t}=-3 D\left(\left(D \Phi_{1}\right)^{2}\right)
$$

whose properties we have studied earlier in detail [12].

From the Lax description, the conserved quantities of the hierarchy can be easily obtained. In fact, the normalized conserved quantities can be written as

$$
\begin{aligned}
H_{n} & =\frac{(-1)^{n+1}}{n} \int d z \operatorname{Res} L^{n} \\
& =\frac{1}{n} \sum_{m=0}^{n}{ }^{n} C_{n-m}{ }^{n-m} C_{m+1} \int d z\left(D \Phi_{0}\right)^{n-2 m-1}\left(D \Phi_{1}\right)^{m+1}
\end{aligned}
$$

Here $d z=d x d \theta$ represents the integration over the superspace. Explicitly, the first few conserved quantities are

$$
\begin{aligned}
H_{1} & =\int d z\left(D \Phi_{1}\right)=0 \\
H_{2} & =\int d z\left(D \Phi_{0}\right)\left(D \Phi_{1}\right) \\
H_{3} & =\int d z\left[\left(D \Phi_{0}\right)^{2}+\left(D \Phi_{1}\right)\right]\left(D \Phi_{1}\right)
\end{aligned}
$$

and so on. As it stands, it is clear that these conserved quantities are fermionic. This is a peculiarity of the sTB-B system (for that matter any -B system) that the Hamiltonians are fermionic. 
Correspondingly, the Hamiltonian structures are odd and we note the first two structures for completeness, namely,

$$
\mathcal{D}_{1}=\left(\begin{array}{ll}
0 & 1 \\
1 & 0
\end{array}\right), \quad \mathcal{D}_{2}=\left(\begin{array}{cc}
2 & D\left(D \Phi_{0}\right) D^{-1} \\
D^{-1}\left(D \Phi_{0}\right) D & D^{-1}\left(D \Phi_{1}\right) D+D\left(D \Phi_{1}\right) D^{-1}
\end{array}\right)
$$

While the Hamiltonian structure $\mathcal{D}_{1}$ can be obtained from the Lax function as the standard GelfandDikii bracket, we do not know how to obtain the second structure from the Lax function. Furthermore, the Jacobi identity for this structure is complicated and needs to be checked. However, these two Hamiltonian structures do lead to the recursion operator

$$
R=\mathcal{D}_{1}^{-1} \mathcal{D}_{2}=\left(\begin{array}{cc}
D^{-1}\left(D \Phi_{0}\right) D & D^{-1}\left(D \Phi_{1}\right) D+D\left(D \Phi_{1}\right) D^{-1} \\
2 & D\left(D \Phi_{0}\right) D^{-1}
\end{array}\right)
$$

which can be easily checked to connect the successive Hamiltonians of the hierarchy.

Supersymmetric integrable systems have conserved non-local charges [20-21] and the dispersionless sTB-B hierarchy also has conserved non-local charges. For example, it can be checked that

$$
\begin{aligned}
Q_{n} & =\frac{(-1)^{n+1}}{n} \int d z\left(D^{-1} \operatorname{Res} L^{n}\right) \\
& =\frac{1}{n} \sum_{m=0}^{n}{ }^{n} C_{n-m}{ }^{n-m} C_{m+1} \int d z\left(D^{-1}\left(\left(D \Phi_{0}\right)^{n-2 m-1}\left(D \Phi_{1}\right)^{m+1}\right)\right)
\end{aligned}
$$

with the first few charges

$$
\begin{aligned}
Q_{1} & =\int d z \Phi_{1} \\
Q_{2} & =\int d z\left(D^{-1}\left(\left(D \Phi_{0}\right)\left(D \Phi_{1}\right)\right)\right) \\
Q_{3} & =\int d z\left(D^{-1}\left(\left(D \Phi_{0}\right)^{2}\left(D \Phi_{1}\right)+\left(D \Phi_{1}\right)^{2}\right)\right)
\end{aligned}
$$

and so on, are conserved under the flow of the system. These are bosonic charges and, interestingly enough, these non-local charges are also related to one another by the same recursion operator $R$ in eq. (18), namely,

$$
\left(\begin{array}{c}
\frac{\delta Q_{n+1}}{\delta \Phi_{0}} \\
\frac{\delta Q_{n+1}}{\delta \Phi_{1}}
\end{array}\right)=R\left(\begin{array}{c}
\frac{\delta Q_{n}}{\delta \Phi_{0}} \\
\frac{\delta Q_{n}}{\delta \Phi_{1}}
\end{array}\right)
$$

It is also nice to see explicitly that if we set $\Phi_{0}=0$ and $\Phi_{1}=\Phi$, then, all the even charges vanish and the odd charges, namely, $H_{2 n+1}$ and $Q_{2 n+1}$ coincide with the corresponding charges of the sKdV-B system [12], as they should. 


\section{Dispersionless Limit of sTB Equation:}

In terms of the same basic variables, $\Phi_{0}$ and $\Phi_{1}$, the sTB hierarchy is described by the Lax operator $[16]$

$$
L=D^{2}-\left(D \Phi_{0}\right)+D^{-1} \Phi_{1},
$$

and the non-standard Lax equation

$$
\frac{\partial L}{\partial t_{n}}=\left[L,\left(L^{n}\right)_{\geq 1}\right]
$$

Explicitly, the lowest order equations have the form

$$
\begin{gathered}
\frac{\partial \Phi_{0}}{\partial t}=-\Phi_{0 x}, \quad \frac{\partial \Phi_{1}}{\partial t}=-\Phi_{1 x} \\
\frac{\partial \Phi_{0}}{\partial t}=-\left(D^{4} \Phi_{0}\right)+D\left(\left(D \Phi_{0}\right)^{2}+2\left(D \Phi_{1}\right)\right) \\
\frac{\partial \Phi_{1}}{\partial t}=\left(D^{4} \Phi_{1}\right)+2 D^{2}\left(\left(D \Phi_{0}\right) \Phi_{1}\right) \\
\frac{\partial \Phi_{0}}{\partial t}=-\left(D^{6} \Phi_{0}\right)+D\left(3 \Phi_{1} \Phi_{0 x}-6\left(D \Phi_{1}\right)\left(D \Phi_{0}\right)-\left(D \Phi_{0}\right)^{3}+3\left(D \Phi_{0}\right)\left(D \Phi_{0 x}\right)\right) \\
\frac{\partial \Phi_{1}}{\partial t}=-\left(D^{6} \Phi_{1}\right)-3 D^{2}\left(\Phi_{1}\left(D \Phi_{0}\right)^{2}+\left(D \Phi_{0}\right) \Phi_{1 x}+\Phi_{1}\left(D \Phi_{1}\right)\right)
\end{gathered}
$$

The last equation allows the reduction $\left(\Phi_{0}=0\right)$ to the supersymmetric KdV equation considered by Manin and Radul [13], namely,

$$
\frac{\partial \Phi_{1}}{\partial t}=-\left(D^{6} \Phi_{1}\right)-3 D^{2}\left(\Phi_{1}\left(D \Phi_{1}\right)\right)
$$

The dispersionless limit of this Lax operator is not as straightforward. However, with some work, it can be determined that the Lax function

$$
L=p-\left(D \Phi_{0}\right)+p^{-1}\left(D \Phi_{1}\right)-p^{-2} \Phi_{0 x} \Phi_{1}+p^{-3} \Phi_{1 x} \Phi_{1}
$$

and the classical Lax equation

$$
\frac{\partial L}{\partial t_{n}}=\left\{\left(L^{n}\right)_{\geq 1}, L\right\}
$$

give the dispersionless sTB hierarchy whose first three equations have the explicit forms

$$
\begin{array}{ccc}
\frac{\partial \Phi_{0}}{\partial t}=-\Phi_{0 x}, & \frac{\partial \Phi_{1}}{\partial t}=-\Phi_{1 x}, \\
\frac{\partial \Phi_{0}}{\partial t}=2\left(D \Phi_{0}\right) \Phi_{0 x}+2 \Phi_{1 x}, & \frac{\partial \Phi_{1}}{\partial t}=2 D^{2}\left(\left(D \Phi_{0}\right) \Phi_{1}\right)
\end{array}
$$




$$
\begin{aligned}
& \frac{\partial \Phi_{0}}{\partial t}=D\left(3 \Phi_{1} \Phi_{0 x}-6\left(D \Phi_{1}\right)\left(D \Phi_{0}\right)-\left(D \Phi_{0}\right)^{3}\right), \\
& \frac{\partial \Phi_{1}}{\partial t}=-3 D^{2}\left(\Phi_{1}\left(D \Phi_{0}\right)^{2}+\Phi_{1}\left(D \Phi_{1}\right)\right) .
\end{aligned}
$$

There are several things to note from here. First, eq. (32), upon setting $\Phi_{0}=0$ and $\Phi_{1}=-\Phi$, gives the dispersionless sKdV equation [12], namely,

$$
\frac{\partial \Phi}{\partial t}=3 D^{2}(\Phi(D \Phi)) \text {. }
$$

This, therefore, gives the non-standard representation of the dispersionless sKdV equation (as opposed to the standard representation given in [12]), and is analogous to the reduction of the sTB hierarchy to the sKdV hierarchy. Second, the normalized conserved quantities of this system can be easily determined to be

$$
\begin{aligned}
H_{n} & =\frac{(-1)^{n+1}}{n} \int d z D^{-1}\left(\operatorname{Res} L^{n}\right) \\
& =\sum_{m=0}^{m_{\max }} \frac{(n-m) !}{m !(m-1) !(n-2 m+1) !} \int d z\left(D \Phi_{0}\right)^{n-2 m+1}\left(D \Phi_{1}\right)^{m-1} \Phi_{1}
\end{aligned}
$$

where the upper limit $m_{\max }=\frac{n}{2}$ if $n$ is even, but $m_{\max }=\left[\frac{n}{2}\right]+1$ if $n$ is odd. The first few of these conserved charges have the explicit forms

$$
\begin{aligned}
H_{1} & =\int d z \Phi_{1} \\
H_{2} & =\int d z\left(D \Phi_{0}\right) \Phi_{1} \\
H_{3} & =\int d z\left(\left(D \Phi_{0}\right)^{2}+\left(D \Phi_{1}\right)\right) \Phi_{1}
\end{aligned}
$$

and so on. These conserved charges are all bosonic and it is clear that if we set $\Phi_{0}=0$ and $\Phi_{1}=\Phi$, all the even charges vanish while the odd charges coincide with those of the dispersionless sKdV hierarchy [12] as they should.

We have not been able to derive the Hamiltonian structures for this system from the GelfandDikii formalism (as is the case in the dispersionless sKdV also[12]). However, the first Hamiltonian structure can be easily checked to be

$$
\mathcal{D}_{1}=\left(\begin{array}{cc}
0 & D \\
D & 0
\end{array}\right)
$$

and this trivially satisfies the Jacobi identity. Notice that this Hamiltonian operator defines a closed skew symmetric two-form

$$
\Omega\left(\mathcal{D}_{1}\right)(a, b)=\int d z\left(a_{1}\left(D b_{2}\right)+a_{2}\left(D b_{1}\right)\right)
$$


where a,b are arbitrary, two component (column matrix) bosonic superfields. We have also checked that it is impossible to construct the second Hamiltonian operator out of local operators alone. However, there is a possibility, which we have not checked, to construct such an operator out of local as well as non-local operators.

Let us now discuss some of the outstanding questions associated with such a system. First, we have not been able to construct the non-local charges from this Lax function. By brute force, we have checked that charges, such as

$$
\begin{aligned}
Q_{n} & =\int d z\left(D^{-1} \Phi_{1}\right)^{n}, \quad n=1,2,3, \cdots \\
Q_{2}^{\prime} & =\int d z\left[\frac{3}{2}\left(D^{-1} \Phi_{1}\right)^{2}+\frac{1}{2} \Phi_{0} \Phi_{1}-\left(D^{-1}\left(\left(D \Phi_{0}\right) \Phi_{1}\right)\right)\right]
\end{aligned}
$$

and so on, are conserved under the flow. Furthermore, under the substitution, $\Phi_{0}=0$ and $\Phi_{1}=\Phi$, these reduce to the appropriate non-local charges of the dispersionless sKdV [12] (In this limit, $\left.Q_{2}=\frac{2}{3} Q_{2}^{\prime}\right)$. However, for lack of a systematic procedure for constructing these charges, we do not have a general expression for the $n$th charge of this set in terms of the Lax function. Furthermore, we do know that the dispersionless sKdV has a second set of non-local charges [12] and since this system can be obtained from the dispersionless sTB, it would be natural to expect the dispersionless sTB also to have a second set of non-local charges. However, we do not know if such a set exists. This emphasizes the need for a systematic understanding of the non-local charges in such systems, described a classical Lax function.

It is also worth recalling that the sTB equation yields the sNLS equation (supersymmetric non-linear Schrödinger equation) under the redefinition [16]

$$
\Phi_{0}=-(D \ln (D Q))+\left(D^{-1}(\bar{Q} Q)\right), \quad \Phi_{1}=-\bar{Q}(D Q)
$$

However, we would like to note here that we have not been able to find a redefinition of fields which would take the dispersionless sTB equation to the dispersionless sNLS equation. In fact, it is worth pointing out that it is not a difficulty only for the supersymmetric system. Even the dispersionless TB equation (bosonic) does not appear to yield the dispersionless NLS equation under the corresponding redefinition. This question certainly needs further study.

\section{$4 \quad N=2$ Formulation for the dispersionless sTB Equation:}

The sTB hierarchy has a $N=2$ supersymmetry [16]. However, it is not manifest in the description of the last section. In order to see the $N=2$ supersymmetry manifestly, we have to define the basic variables of the theory appropriately. Let us recall that in the conventional description of the system (as given in the previous section), the basic variables were two fermionic superfields which depended on the usual bosonic coordinates, $(x, t)$, but they also depended on an additional 
anti-commuting Grassmann variable $\theta$. The Taylor expansion of such superfields in the Grassmann coordinate is simple and has been given in eq. (1). In the presence of an $N=2$ supersymmetry, the superfields depend on $(x, t)$ as well as two anti-commuting variables $\theta_{1}$ and $\theta_{2}$. (Incidentally, an $N=2$ supersymmetric system can also be described on an $N=1$ superspace which is the description used in the earlier section.) Expanding the superfield $\Phi\left(x, t, \theta_{1}, \theta_{2}\right)$ in a Taylor series in the anti-commuting variables, we obtain

$$
\Phi=\phi_{1}+\theta_{1} \chi_{1}+\theta_{2} \chi_{2}+\theta_{2} \theta_{1} \phi_{2}
$$

where $\phi_{1}, \phi_{2}$ are bosonic functions (or fermionic) and $\chi_{1}, \chi_{2}$ are fermionic (or bosonic) if $\Phi$ is a bosonic (or fermionic) superfield. In this case, the super-covariant derivatives on the $N=2$ superspace are defined as

$$
\begin{aligned}
& D_{1}=\frac{\partial}{\partial \theta_{1}}+\theta_{1} \frac{\partial}{\partial x}, \quad D_{2}=\frac{\partial}{\partial \theta_{2}}+\theta_{2} \frac{\partial}{\partial x} \\
& D_{1}^{2}=D_{2}^{2}=\partial=\frac{\partial}{\partial x}, \quad D_{2} D_{1}=-D_{1} D_{2} .
\end{aligned}
$$

Recently several methods have been proposed to obtain $N=2$ supersymmetric soliton equations [22-25]. In this paper, we consider two different supersymmetric Lax operators which generate two distinct sTB hierarchies. The first is connected with the $N=2, a=1[22,24]$ supersymmetric $\mathrm{KdV}$ equation (namely, it reduces to this model upon appropriate redefinition) and is defined by the Lax operator

$$
L=\partial+\tilde{\Phi}_{0}+\partial^{-1} D_{1} D_{2} \tilde{\Phi}_{1} .
$$

where $\tilde{\Phi}_{0}$ and $\tilde{\Phi}_{1}$ are two bosonic superfields (and we have used a tilde to avoid confusion with the superfields of the earlier sections). This Lax operator is related to the supersymmetric Lax operator considered in [24], through the gauge transformation

$$
L=e^{-g} L e^{g},
$$

where $g=\int d x d \theta_{1} d \theta_{2} G=\int d Z G$ with $G$ a bosonic superfield.

It is straightforward to check that the Lax operator of eq. (42) leads to consistent dynamical equations through the nonstandard Lax relation

$$
\frac{\partial L}{\partial t_{n}}=\left[L,\left(L^{n}\right)_{\geq 1}\right]
$$

Explicitly, we can write the first three flows as

$$
\begin{gathered}
\frac{\partial \tilde{\Phi}_{0}}{\partial t}=-\tilde{\Phi}_{0 x}, \quad \frac{\partial \tilde{\Phi}_{1}}{\partial t}=-\tilde{\Phi}_{1 x} \\
\frac{\partial \tilde{\Phi}_{0}}{\partial t}=\partial\left(\tilde{\Phi}_{0 x}-\tilde{\Phi}_{0}^{2}-2\left(D_{1} D_{2} \tilde{\Phi}_{1}\right)+\tilde{\Phi}_{1}^{2}\right), \quad \frac{\partial \tilde{\Phi}_{1}}{\partial t}=\partial\left(\tilde{\Phi}_{1 x}-2 \tilde{\Phi}_{1} \tilde{\Phi}_{0}\right)
\end{gathered}
$$




$$
\begin{aligned}
& \frac{\partial \tilde{\Phi}_{0}}{\partial t}=\partial\left(-\tilde{\Phi}_{0 x x}-3 \tilde{\Phi}_{o x} \tilde{\Phi}_{0}-\tilde{\Phi}_{0}^{3}-3\left(D_{1} D_{2} \tilde{\Phi}_{1}\right) \tilde{\Phi}_{0}+3 \tilde{\Phi}_{1}^{2} \tilde{\Phi}_{0}-3\left(D_{1} D_{2}\left(\tilde{\Phi}_{1} \tilde{\Phi}_{0}\right)\right)\right) \\
& \frac{\partial \tilde{\Phi}_{1}}{\partial t}=\partial\left(-\tilde{\Phi}_{1 x x}-3\left(D_{1} D_{2} \tilde{\Phi}_{1}\right) \tilde{\Phi}_{1}+\tilde{\Phi}_{1}^{3}+3 \tilde{\Phi}_{1 x} \tilde{\Phi}_{0}-3 \tilde{\Phi}_{1} \tilde{\Phi}_{0}^{2}\right) .
\end{aligned}
$$

The last equation leads, upon the reduction $\tilde{\Phi}_{0}=0$, to the $N=2, a=1[22]$ supersymmetric $\mathrm{KdV}$ equation

$$
\frac{\partial \tilde{\Phi}_{1}}{\partial t}=\partial\left(-\tilde{\Phi}_{1 x x}-3\left(D_{1} D_{2} \tilde{\Phi}_{1}\right) \tilde{\Phi}_{1}+\tilde{\Phi}_{1}^{3}\right)
$$

We can also construct a second Lax operator of the form

$$
L=D_{1}+\bar{\Phi}_{0}+\partial^{-1} D_{2} \bar{\Phi}_{1}
$$

where $\bar{\Phi}_{0}$ is a fermionic superfield while $\bar{\Phi}_{1}$ is a bosonic superfield. Note that the Lax operator, in the present case, is fermionic while that in eq. (42) was bosonic. Nonetheless, as in the previous case, it is easy to check that this Lax operator is gauge equivalent to the one considered in [25]. This Lax operator leads to dynamical equations of the non-standard form

$$
\frac{\partial L}{\partial t_{2 n}}=\left[L,\left(L^{2 n}\right)_{\geq 1}\right]
$$

Explicitly, the first three flows of this hierarchy have the forms

$$
\begin{gathered}
\frac{\partial \bar{\Phi}_{0}}{\partial t}=-\bar{\Phi}_{0 x}, \quad \frac{\partial \bar{\Phi}_{1}}{\partial t}=-\bar{\Phi}_{1 x}, \\
\frac{\partial \bar{\Phi}_{0}}{\partial t}=D_{1}\left(-\left(D_{1} \bar{\Phi}_{0 x}\right)-2\left(D_{1} D_{2} \bar{\Phi}_{1}\right)-2\left(D_{1} \bar{\Phi}_{0}\right)^{2}-2 \bar{\Phi}_{1}^{2}-\bar{\Phi}_{1}\left(D_{2} \bar{\Phi}_{0}\right)\right), \\
\frac{\partial \bar{\Phi}_{1}}{\partial t}=D_{1}\left(\left(D_{1} \bar{\Phi}_{1 x}\right)-2 \bar{\Phi}_{0 x} \bar{\Phi}_{1}-\left(D_{2} \bar{\Phi}_{1}\right) \bar{\Phi}_{1}-2\left(D_{1} \bar{\Phi}_{1}\right)\left(D_{1} \bar{\Phi}_{0}\right)\right), \\
\left.\frac{\partial \bar{\Phi}_{0}=D_{1}\left(-\left(D_{1} \bar{\Phi}_{0 x x}-3\left(D_{1} \bar{\Phi}_{0 x}\right)\left(D_{1} \bar{\Phi}_{0}\right)-6\left(D_{1} D_{2} \bar{\Phi}_{1}\right)\left(D_{1} \bar{\Phi}_{0}\right)-6 \bar{\Phi}_{1}^{2}\left(D_{1} \bar{\Phi}_{0}\right)\right.\right.}{\partial t}=\bar{\Phi}_{1} \bar{\Phi}_{1}=D_{2} \bar{\Phi}_{0}\right)\left(D_{1} \bar{\Phi}_{0}\right)+3\left(D_{2} \bar{\Phi}_{1} \bar{\Phi}_{0 x}+3\left(D_{1} \bar{\Phi}_{1}\right)\left(D_{1} D_{2} \bar{\Phi}_{0}\right)-\left(D_{1} \bar{\Phi}_{0}\right)^{3}\right), \\
-6\left(D_{2} \bar{\Phi}_{1}\right) \bar{\Phi}_{1}\left(D_{1} \bar{\Phi}_{0}\right)+3\left(D_{1} \bar{\Phi}_{1 x}\right)\left(D_{1} \bar{\Phi}_{0}\right)-3\left(D_{1} \bar{\Phi}_{1}\right)\left(D_{1} \bar{\Phi}_{0}\right)^{2} \\
\left.-3\left(D_{1} \bar{\Phi}_{1}\right)\left(D_{1} D_{2} \bar{\Phi}_{1}\right)-6\left(D_{1} \bar{\Phi}_{1}\right) \bar{\Phi}_{1}^{2}-6\left(D_{1} \bar{\Phi}_{1}\right) \bar{\Phi}_{1}\left(D_{2} \bar{\Phi}_{0}\right)\right)
\end{gathered}
$$

Note that when $\bar{\Phi}_{0}=0$, the last equation reduces to the $N=2, a=-2[22]$ supersymmetric $\mathrm{KdV}$ equation. Unfortunately the Lax operator, which gives rise to the sTB hierarchy that contains the $N=2, a=4$ [22] supersymmetric $\mathrm{KdV}$ equation is not known as yet. Furthermore, since fermionic Lax operators do not lend easily to a dispersionless limit, we will not discuss this system any further. 
In order to obtain the dispersionless sTB hierarchy, we now have to introduce the concept of the fermionic momenta on the $N=2$ superspace. There will be two such fermionic momenta defined by $[12,26]$

$$
\Pi_{1}=-\left(p_{\theta_{1}}+\theta_{1} p\right), \quad \Pi_{2}=-\left(p_{\theta_{2}}+\theta_{2} p\right),
$$

We can now assume the "commutation" rules for the functions $\Pi_{i}$ as

$$
\left\{\Pi_{i}, \Pi_{j}\right\}=-2 p \delta_{i j}
$$

Note that the $\Pi_{i}$ 's generate covariant differentiation through the PB relation (for any superfield $A$ )

$$
\left\{\Pi_{i}, A\right\}=\left(D_{i} A\right), \quad i=1,2 .
$$

In trying to obtain the dispersionless $N=2$ sTB hierarchy, we start from the Lax operator for the $N=2$ sTB hierarchy in eq. (42), and assume the Lax function for the dispersionless system to have the general form

$$
L=p+k_{1} \Pi_{1} \Pi_{2}+\tilde{\Phi}_{0}+\sum_{s=1}^{3} p^{-s}\left(F_{0}^{s}+\Pi_{1} F_{1}^{s}+\Pi_{2} F_{2}^{s}+\Pi_{1} \Pi_{2} F_{3}^{s}\right) .
$$

where $k_{1}$ is an arbitrary coefficient and $F_{k}^{s}, k=0,1,2,3, s=1,2,3$ are arbitrary functions of $\tilde{\Phi}_{0}$ and $\tilde{\Phi}_{1}$.

We have checked that the classical analogue of eq. (44), namely,

$$
\frac{\partial L}{\partial t_{n}}=\left\{\left(L^{n}\right)_{\geq 1}, L\right\}
$$

with the projection $\geq 1$ defined as

$$
\begin{aligned}
& \left(\sum_{s=-\infty}^{\infty} p^{s}\left(F_{0}^{s}+\Pi_{1} F_{1}^{s}+\Pi_{2} F_{2}^{s}+\Pi_{1} \Pi_{2} F_{3}^{3}\right)\right)_{\geq 1}= \\
& \Pi_{1} F_{1}^{s}+\Pi_{2} F_{2}^{s}+\Pi_{1} \Pi_{2} F_{3}^{s}+\sum_{s=1}^{\infty} p^{s}\left(F_{0}^{s}+\Pi_{1} F_{1}^{s}+\Pi_{2} F_{2}^{s}+\Pi_{1} \Pi_{2} F_{3}^{3}\right) .
\end{aligned}
$$

leads to two possible solutions with $k_{1}=0$ in either case.

The first Lax function that leads to consistent equations has the form

$$
L=p+\tilde{\Phi}_{0}+p^{-1} \Pi_{1} \Pi_{2} \tilde{\Phi}_{1}
$$

The first three flows of this hierarchy have the explicit forms

$$
\begin{array}{rlrl}
\frac{\partial \tilde{\Phi}_{0}}{\partial t} & =-\tilde{\Phi}_{0 x}, & & \frac{\partial \tilde{\Phi}_{1}}{\partial t}=-\tilde{\Phi}_{1 x}, \\
\frac{\partial \tilde{\Phi}_{0}}{\partial t}=\partial\left(\tilde{\Phi}_{0}^{2}\right), & \frac{\partial \tilde{\Phi}_{1}}{\partial t}=2 \partial\left(\tilde{\Phi}_{1} \tilde{\Phi}_{0}\right) \\
\frac{\partial \tilde{\Phi}_{0}}{\partial t}=\partial\left(\tilde{\Phi}_{0}^{3}\right), & \frac{\partial \tilde{\Phi}_{1}}{\partial t}=3 \partial\left(\tilde{\Phi}_{1} \tilde{\Phi}_{0}^{2}\right)
\end{array}
$$


However, this hierarchy appears trivial since the equations do not have any explicit dependence on supersymmetric covariant derivatives. Therefore, we will not consider this hierarchy any further.

The second Lax function does not contain the fermionic functions $\Pi_{i}$ and has the form

$$
L=p+\tilde{\Phi}_{0}+p^{-1}\left(D_{1} D_{2} \tilde{\Phi}_{1}\right)-p^{-2}\left(D_{2} \tilde{\Phi}_{1}\right)\left(D_{1} \tilde{\Phi}_{0}\right)+p^{-3}\left(D_{2} \tilde{\Phi}_{1 x}\right)\left(D_{2} \tilde{\Phi}_{1}\right)
$$

This, on the other hand, produces an interesting supersymmetric hierarchy whose first three flows have the forms

$$
\begin{gathered}
\frac{\partial \tilde{\Phi}_{0}}{\partial t}=-\tilde{\Phi}_{0 x}, \quad \frac{\partial \tilde{\Phi}_{1}}{\partial t}=-\tilde{\Phi}_{1 x}, \\
\frac{\partial \tilde{\Phi}_{0}}{\partial t}=\partial\left(2\left(D_{1} D_{2} \tilde{\Phi}_{1}\right)+\tilde{\Phi}_{0}^{2}\right), \\
\frac{\partial \tilde{\Phi}_{1}}{\partial t}=2 D_{2}\left(\left(D_{2} \tilde{\Phi}_{1}\right) \tilde{\Phi}_{0}\right) \\
\frac{\partial \tilde{\Phi}_{0}}{\partial t}=\partial\left(\tilde{\Phi}_{0}^{3}+6\left(D_{1} D_{2} \tilde{\Phi}_{1}\right) \tilde{\Phi}_{0}-3\left(D_{2} \tilde{\Phi}_{1}\right)\left(D_{1} \tilde{\Phi}_{0}\right)\right), \\
\frac{\partial \tilde{\Phi}_{1}}{\partial t}=3 D_{2}\left(\left(D_{2} \tilde{\Phi}_{1}\right) \tilde{\Phi}_{0}^{2}+\left(D_{2} \tilde{\Phi}_{1}\right)\left(D_{1} D_{2} \tilde{\Phi}_{1}\right)\right) .
\end{gathered}
$$

It is interesting to note that, when $\tilde{\Phi}_{0}=0$, the last equation becomes

$$
\frac{\partial \tilde{\Phi}_{1}}{\partial t}=3 D_{2}\left(\left(D_{2} \tilde{\Phi}_{1}\right)\left(D_{1} D_{2} \tilde{\Phi}_{1}\right)\right) .
$$

which, in fact, reduces to eq. (33) with the substitution $\left.\left(D_{2} \tilde{\Phi}_{1}\right)\right|_{\theta_{2}=0}=-\Phi_{1}$. Therefore, eq. (66) can be considered as the $N=2$ generalization of the dispersionless $N=1$ supersymmetric KdV-MR equation.

Let us also note that the Lax function in eq. (62) as well as the resulting hierarchy coincide with those of the previous section with the identifications

$$
\left.\left(D_{2} \tilde{\Phi}_{1}\right)\right|_{\theta_{2}=0}=-\Phi_{1},\left.\quad \tilde{\Phi}_{0}\right|_{\theta_{2}=0}=\left(D_{1} \Phi_{0}\right)=-\left(D \Phi_{0}\right) .
$$

Namely, the redefinition also involves $\theta_{1} \rightarrow-\theta$ or equivalently, $D_{1} \rightarrow-D$. Notice that these transformations are highly nontrivial reductions. Consequently, we do not expect the conserved quantities of eqs. (34) to define conserved quantities of the $N=2$ supersymmetric hierarchy and we have verified this. On the other hand, we can construct conserved quantities for the $N=2$ hierarchy directly and we have found two such series of conserved quantities, by brute force, using the computer.

The first set consists of bosonic conserved charges of the form

$$
\begin{aligned}
H_{n} & =\int d Z\left(\tilde{\Phi}_{1}\right)^{n}, & n=1,2,3,4, \ldots \\
\tilde{H}_{1} & =\int d Z \tilde{\Phi}_{0} & \\
\tilde{H}_{2} & =\int d Z \tilde{\Phi}_{1} \tilde{\Phi}_{0} &
\end{aligned}
$$


where $d Z=d x d \theta_{1} d \theta_{2}$. The second series of conserved charges is fermionic of the form

$$
\begin{aligned}
H_{5 / 2}= & \int d Z\left(D_{2} \tilde{\Phi}_{1}\right) \tilde{\Phi}_{0} \\
H_{7 / 2}= & \int d Z\left(D_{2} \tilde{\Phi}_{1}\right)\left(\tilde{\Phi}_{0}^{2}+\left(D_{1} D_{2} \tilde{\Phi}_{1}\right)\right), \\
H_{9 / 2}= & \int d Z\left(D_{2} \tilde{\Phi}_{1}\right)\left(3\left(D_{1} D_{2} \tilde{\Phi}_{1}\right) \tilde{\Phi}_{0}+\tilde{\Phi}_{0}^{3}\right), \\
H_{11 / 2}= & \int d Z\left(4\left(D_{2} \tilde{\Phi}_{1 x}\right) \tilde{\Phi}_{1 x} \tilde{\Phi}_{1}-4\left(D_{2} \tilde{\Phi}_{1}\right) \tilde{\Phi}_{1 x x} \tilde{\Phi}_{1}+12\left(D_{1} \tilde{\Phi}_{1 x}\right)\left(D_{1} D_{2} \tilde{\Phi}_{1}\right) \tilde{\Phi}_{1}\right. \\
& \left.\quad+18\left(D_{2} \tilde{\Phi}_{1}\right)\left(D_{1} D_{2} \tilde{\Phi}_{1}\right) \tilde{\Phi}_{0}^{2}+3\left(D_{2} \tilde{\Phi}_{1}\right) \tilde{\Phi}_{0}^{4}\right)
\end{aligned}
$$

Here, we have labelled the conserved quantities by the weights of the integrand $\left(\left[\tilde{\Phi}_{0}\right]=1=\left[\tilde{\Phi}_{1}\right]\right.$ and $\left.\left[D_{1}\right]=\frac{1}{2}=\left[D_{2}\right]\right)$. Note that the second set of charges in eq. (69) follows from the Lax function, up to non-essential normalization, as

$$
H_{n+\frac{1}{2}}=\int d Z D_{1}^{-1} \operatorname{Res} L^{n}
$$

However, we do not know how to obtain the first set (except for the lowest one) from the Lax function, nor is it clear that these exhaust all the conserved charges of the system.

Comparing with the discussion of the previous section and particularly from the form of the non-local conserved charges in eq. (38), we see that we can write non-local conserved charges for the $N=2$ system as

$$
\tilde{Q}_{n}=\int d Z\left(D_{1}^{-1} D_{2} \tilde{\Phi}_{1}\right)^{n} \quad n=1,2,3,4 \cdots
$$

It is, in fact, quite straightforward to check that they are conserved. Similarly, we note that

$$
\tilde{Q}_{2}^{\prime}=\int d Z\left(D_{1}^{-1}\left(\tilde{\Phi}_{0}\left(D_{2} \tilde{\Phi}_{1}\right)\right)-\frac{1}{2}\left(D_{1}^{-1} \tilde{\Phi}_{0}\right)\left(D_{2} \tilde{\Phi}_{1}\right)-\frac{3}{2} \tilde{\Phi}_{1}^{2}\right)
$$

also represents a conserved charge (compare with the second of the charges in eq. (38)). However, we do not know how to obtain these from the Lax function directly.

The first Hamiltonian structure for the dispersionless $N=2$ hierarchy of eqs. (63)-(69) is easily seen to be

$$
\tilde{\mathcal{D}}_{1}=\left(\begin{array}{cc}
0 & D_{2} \\
-D_{2} & 0
\end{array}\right)
$$

and is trivially seen to satisfy the Jacobi identity. This Hamiltonian operator defines the closed skew symmetric two-form

$$
\Omega\left(\tilde{\mathcal{D}}_{1}\right)(a, b)=\int d Z\left(a_{1}\left(D b_{2}\right)-a_{2}\left(D b_{1}\right)\right)
$$

where in contrast to the $N=1$ case $a$ and $b$ are arbitrary, two component fermionic superfields. 


\section{Conclusion}

In this paper, we have studied the dispersionless limits of the sTB-B, the sTB as well as the $N=2$ sTB hierarchies in detail. We have obtained the Lax descriptions in terms of classical Lax functions, obtained conserved local as well as some of the non-local charges and brought out various other features associated with such systems. We have also tried to point out various open questions associated with such systems, the most pressing of which is a systematic understanding of the construction of non-local charges for such systems starting from the Lax description as well as a

generalization of the Gelfand-Dikii procedure for construction of Hamiltonian structures for such systems..

\section{Acknowledgments}

A.D. acknowledges support in part by the U.S. Dept. of Energy Grant DE-FG 02-91ER40685 while Z.P. is supported in part by the Polish KBN Grant 2 P0 3B 13616.

\section{References}

[1] Yu.I. Manin, J. Sov. Math. 11 (1979) 1.

[2] B.A. Dubrovin and S.P. Novikov, Russian Math. Surveys 44 (1989) 35.

[3] V.E. Zakharov, Funct. Anal. Appl. 14 (1980) 89.

[4] J. Cavalcante and H.P. McKean, Physica 4D (1982) 253.

[5] P.J. Olver and Y. Nutku, J. Math. Phys. 29 (1988) 1610.

[6] G.B. Whitham, Linear and Nonlinear Waves, John Wiley, 1974.

[7] J.C. Brunelli, Rev. Math. Phys. 8 (1996) 1041.

[8] J.C. Brunelli and A. Das, Phys. Lett. A235 (1997) 597.

[9] M. Arik, F. Neyzi, Y. Nutku, P.J. Olver and J. Verosky, J. Math. Phys. 30 (1988) 1338.

[10] J.C. Brunelli and A. Das, Phys. Lett. B426 (1998) 57.

[11] D. Lebedev and Yu.I. Manin, Phys. Lett. 74A (1979) 154; V.E. Zakharov, Physica 3D (1981) 193; Y. Kodama and J. Gibbons, Phys. Lett. 135A (1989) 167; I.M. Krichever, Comm. Math. Phys. 143 (1991) 415; K. Takasaki and T. Takebe, Rev. Math. Phys. 7 (1995) 743; A.M. Bloch, H. Flaschka and T. Ratiu, Comm. Fields Inst. 7 (1995) 57. 
[12] J. Barcelos-Neto, A. Constandache and A. Das, solv-int/9910001.

[13] Yu. I. Manin and A. O. Radul, Comm. Math. Phys. 98 (1985) 65; P. Mathieu, Phys. Lett. B203 (1988) 287; P. Mathieu, J. Math. Phys. 29 (1988) 2499.

[14] B.A. Kupershmidt, Phys. Lett. A102 (1984) 213.

[15] B. Kupershmidt, Comm. Math. Phys. 99 (1985) 51; L. J. F. Broer, Appl. Sci. Res. 31 (1975) 377; D. J. Kaup, Prog. Theo. Phys. 54 (1975) 396.

[16] J. C. Brunelli and A. Das, Phys. Lett. B337 (1994) 303; J. C. Brunelli and A. Das, Int. J. Mod. Phys. A10 (1995) 4563.

[17] F. Toppan, Int. J. Mod. Phys. A10 (1995) 895; S. Krivonos and A. Sorin, Phys.Lett B357 (1995) 94; S. Krivonos, A. Sorin and F. Toppan, Phys.Lett A206 (1995) 146.

[18] J. C. Brunelli and A. Das, Phys. Lett. B409 (1997) 229.

[19] K. Becker and M. Becker, Mod. Phys. Lett. A8 (1993) 1205.

[20] P. Dargis and P. Mathieu, Phys. Lett. A176 (1993) 67.

[21] J.C. Brunelli and A. Das, Phys. Lett. B354 (1995) 307.

[22] P.Laberge and P.Mathieu Phys.Lett B215 (1988) 718; P.Labelle and P.Mathieu J.Math.Phys. 32 (1991) 923.

[23] Z.Popowicz Phys. Lett. B459 (1999) 150.

[24] Z.Popowicz J.Phys. A29 (1996) 4987; Z.Popowicz Phys.Lett A174 (1993) 411.

[25] F.Delduc, L.Gallot and A.Sorin Nucl.Phys B558 (1999) 535.

[26] For a general review of phase space structure of such supersymmetric theories, see, for example, J. Barcelos-Neto and A. Das, Phys. Rev. D33 (1986) 2863.

[27] A.Hearn Reduce "User's Manula 3.7" (1999).

[28] Z.Popowicz Compt.Phys.Commun 100 (1997) 277. 